\title{
Determination of Fortified Rosin-Glycerin Ester Sizing Agents in Paper by Reactive Pyrolysis-Gas Chromatography in the Presence of an Organic Alkali
}

\author{
Lili Wang, Yasuyuki IshIDA, Hajime OHTAni and Shin Tsuge ${ }^{\dagger}$ \\ Department of Applied Chemistry, Graduate School of Engineering, Nagoya University, \\ Nagoya 464-8603, Japan
}

Keywords Pyrolysis-gas chromatography, rosin-glycerin ester sizing agent, reactive pyrolysis, tetramethylammonium hydroxide

Rosin is one of the most popular and traditional sizing agents which are often used in the paper-making process to increase the water-resistance of printing and writing paper. Recently, fortified rosins consisting of rosin acid and its adducts have been prepared by a partial reaction of rosin acid with maleic anhydride or fumaric acid, and widely utilized in practical paper manufacturing. More recently, a fortified rosin-glycerin ester sizing agent has been developed as one of the most promising sizing agents to obtain not only improved sizing efficiency, but also better lubricability of the surface of paper during the papermaking process. Figure 1 shows a typical preparation route of the fortified rosin-glycerin ester sizing agent, which consists of a mixture of the rosin-glycerin ester and the rosin adduct-glycerin ester.

It is important to determine the amount of sizing agent retained in paper samples for their quality control, because the rosin emulsion added to pulp slurry is partly lost with white water during paper production. Usually, the amounts of rosin sizing agent retained in paper is determined by extracting the rosin with some organic solvent, followed by weighing the extract after evaporating the solvent. ${ }^{1}$ Another method to determine the rosin content using the absorbance of the extract is also reported. ${ }^{2}$ However, even the time-consuming extraction procedure often results in an insufficient recovery, causing a less-accurate determination because trace amounts of the rosin components are not only physically retained in the paper, but are also chemically bound to the paper matrix to some extent after fixing.

One of the most representative techniques used to determine various trace sizing agents in paper is pyrolysis-gas chromatography (Py-GC), which has been successfully applied to the analysis of wet-strength resin ${ }^{3}$ and neutral sizing agents. ${ }^{4}$ The conventional Py-GC, however, is not directly applicable to determine rosin

† To whom correspondence should be addressed. sizing agents because of their extremely high polarities, even using a high-resolution capillary column. Recently, reactive pyrolysis in the presence of an organic alkali, tetramethylammonium hydroxide (TMAH) $\left(\left(\mathrm{CH}_{3}\right)_{4} \mathrm{NOH}\right)$, was successfully applied to determine the amounts of the rosin sizing agents in paper samples. ${ }^{5}$

In this work, reactive Py-GC in the presence of organic alkali was extended in order to determine the fortified rosin-glycerin ester sizing agents in paper samples.

\section{Experimental}

\section{Materials}

The five paper samples (P-1 - P-5) used in this work are listed in Table 1. These samples, containing various amounts of the fortified rosin-glycerin ester sizing agent, were prepared by adding the sizing agent and other additives, such as alum $\left(\mathrm{Al}_{2}\left(\mathrm{SO}_{4}\right)_{3} \cdot 18 \mathrm{H}_{2} \mathrm{O}\right)$ $1.0 \% \mathrm{~m} / \mathrm{m}, \mathrm{CaCO}_{3} 10 \% \mathrm{~m} / \mathrm{m}$ and cationic starch $1.0 \% \mathrm{~m} / \mathrm{m}$ to the pulp slurry, under conditions similar to those for commercial paper production. A series of standard samples for the calibration were also prepared using a blank paper sample (P-1) produced without adding any sizing agent. In preparing these standard

Table 1 Paper samples containing fortified rosin-glycerin ester sizing agents

\begin{tabular}{cc}
\hline Sample & $\begin{array}{c}\text { Rosin-glycerin ester } \\
\text { sizing agent }(\% \mathrm{~m} / \mathrm{m}) \text { added }\end{array}$ \\
\hline P-1 & 0 \\
P-2 & 0.1 \\
P-3 & 0.3 \\
P-4 & 0.5 \\
P-5 & 1.0 \\
\hline
\end{tabular}



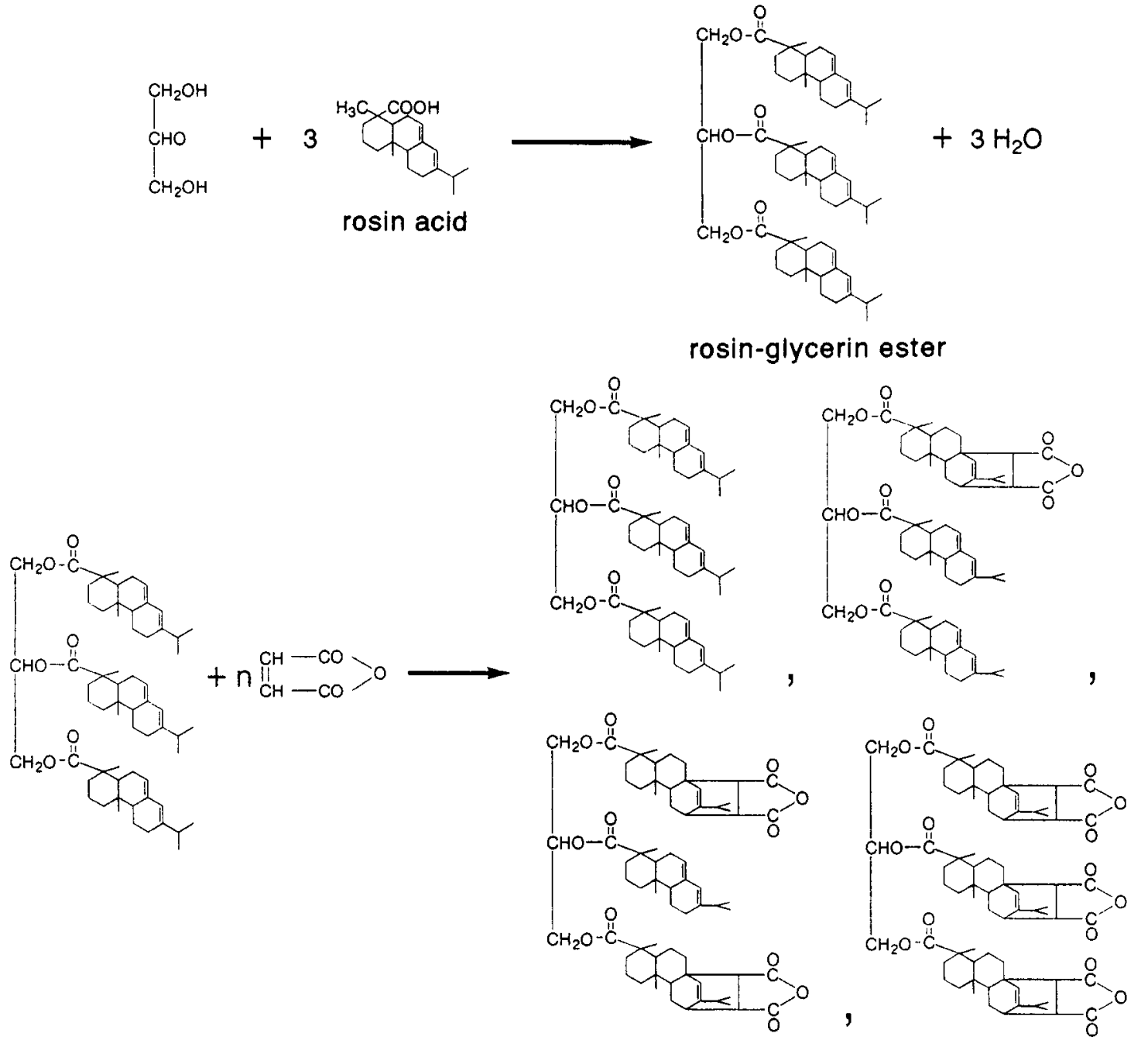

fortified rosin-glycerin ester

Fig. 1 Preparation of a fortified rosin-glycerin ester sizing agent.

samples, a known amount of the sizing agent dissolved in a known volume of acetone was added directly to a weighed piece of blank paper with a microsyringe, and then dried in vacuo. The thus-prepared paper samples were cryo-milled into a fine powder (less than 60 mesh) by a freezer mill (SPEX 6700) prior to Py-GC measurements in order to homogenize them.

Tetramethylammonium hydroxide (TMAH, $25 \% \mathrm{~m} / \mathrm{m}$ solution in methanol) supplied by Aldrich Chemical Company, Inc. was used as received.

\section{Apparatus}

A vertical microfurnace pyrolyzer (Yanaco GP-1018) was directly attached to a gas chromatograph (HP 5890) equipped with a flame-ionization detector (FID). A fused-silica capillary column (HP Ultra 1, $25 \mathrm{~m} \times 0.20$ $\mathrm{mm}$ i.d. coated with $0.33 \mu \mathrm{m}$ film of crosslinked methylsilicone) was used. An aliquot of a finely powdered paper sample weighing $c a$. $500 \mu \mathrm{g}$, or rosin glycerin ester alone weighing $c a .50 \mu \mathrm{g}$, was placed in a platinum sample cup together with approximately $2 \mu \mathrm{l}$ of the TMAH solution, and then pyrolyzed at $400^{\circ} \mathrm{C}$ in a stream of He carrier gas $\left(50 \mathrm{ml} \mathrm{min}{ }^{-1}\right)$, the flow rate of which was reduced to $1 \mathrm{ml} / \mathrm{min}$ at the capillary separation column by means of a splitter. The column temperature was programmed from 50 to $300^{\circ} \mathrm{C}$ at a rate of $8^{\circ} \mathrm{C} \mathrm{min}^{-1}$. Identification of the peaks on the pyrograms was mainly performed by a GC-MS (JEOL AUTOMASS 150), to which a microfurnace pyrolyzer (Frontier Lab Single Shot Pyrolyzer PY-2010SL) was also attached.

\section{Results and Discussion}

Role of the organic alkali reagent in the pyrolysis of rosin-glycerin ester sizing agent in paper sample

Figure 2 shows the pyrograms obtained for the fortified rosin-glycerin ester sizing agent alone without the addition of TMAH (a) and with the addition of TMAH (b) at $400^{\circ} \mathrm{C}$. Although a series of relatively weak peaks of rosin acids were observed at between 20 and 
(a)

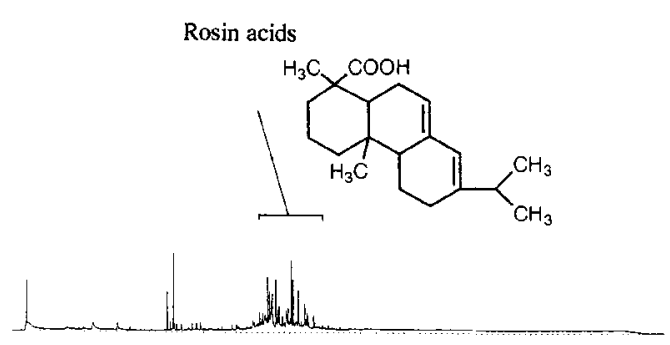

(b)

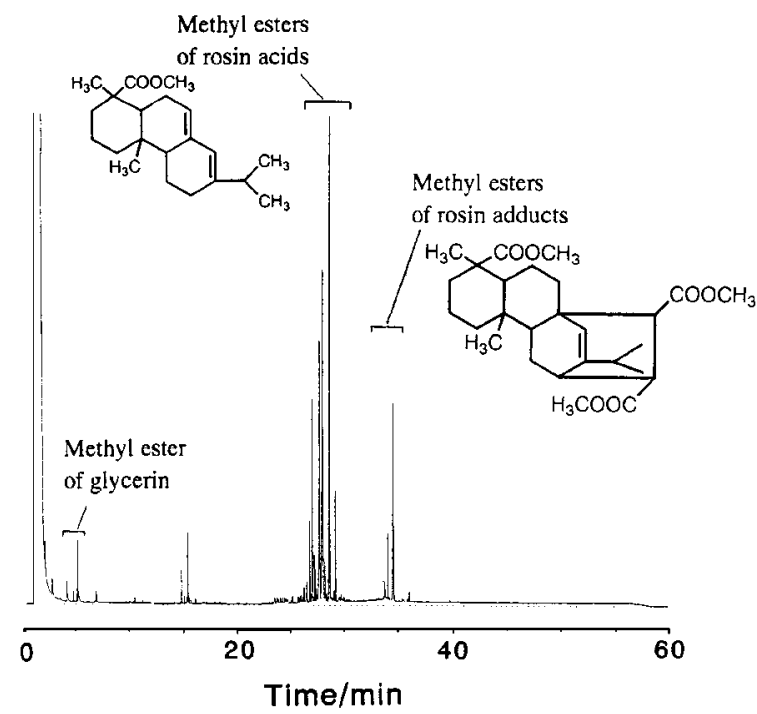

Fig. 2 Pyrograms of a rosin-glycerin ester sizing agent at $400^{\circ} \mathrm{C}$ without TMAH (a) and with TMAH (b).

$25 \mathrm{~min}$ in the pyrogram of Fig. 2(a), no peaks of the rosin adducts were observed, because of their extremely high polarity. On the contrary, in the pyrogram obtained in the presence of TMAH (Fig. 2(b)), two groups of sharp peaks of the methyl esters of rosin acids and their adducts were clearly observed at between 25 and $30 \mathrm{~min}$, and 33 and $37 \mathrm{~min}$, respectively, as a result of the selective hydrolysis of ester bondings, followed by the simultaneous methylation of the carboxyl groups. In addition, the peaks of the methyl ethers of glycerin were also observed at around $5 \mathrm{~min}$. These results suggest that reactive pyrolysis in the presence of TMAH could be applied to determine the fortified rosin-glycerin ester sizing agent in paper samples.

Figure 3 shows pyrograms obtained for a paper sample (P-5) prepared by adding $1 \% \mathrm{~m} / \mathrm{m}$ of the sizing agent without the addition of TMAH (a) and with the addition of TMAH (b), and that of a blank paper sample (P-1) with the addition of TMAH (c) at $400^{\circ} \mathrm{C}$. From these results, it has been found that the reactive pyrolysis of a paper sample containing the rosin glycerin ester can produce very sharp derivatized peaks, not only for rosin acids, but also for rosin adducts. However, the peaks of the methyl ester of rosin acids somewhat overlap those of disaccharides formed from the pulp matrix. Moreover, there exist possibilities that (a)

(b)

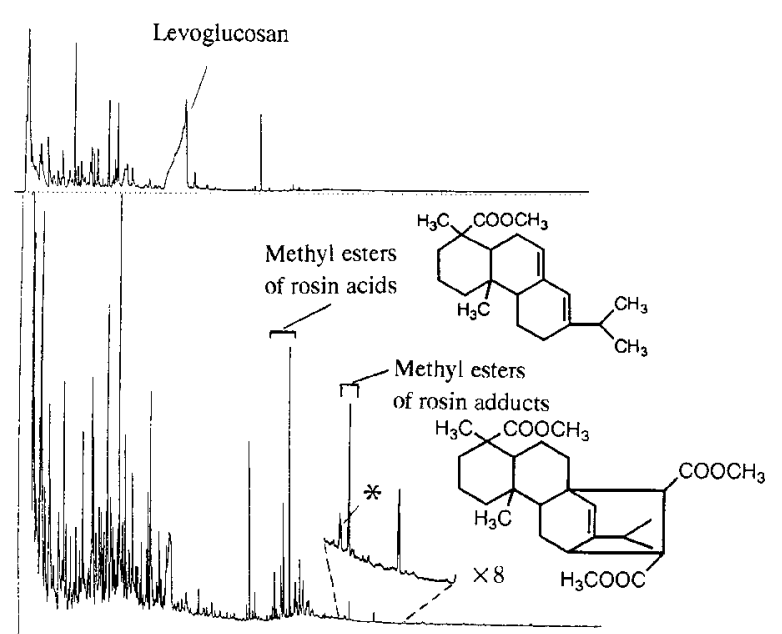

(c)

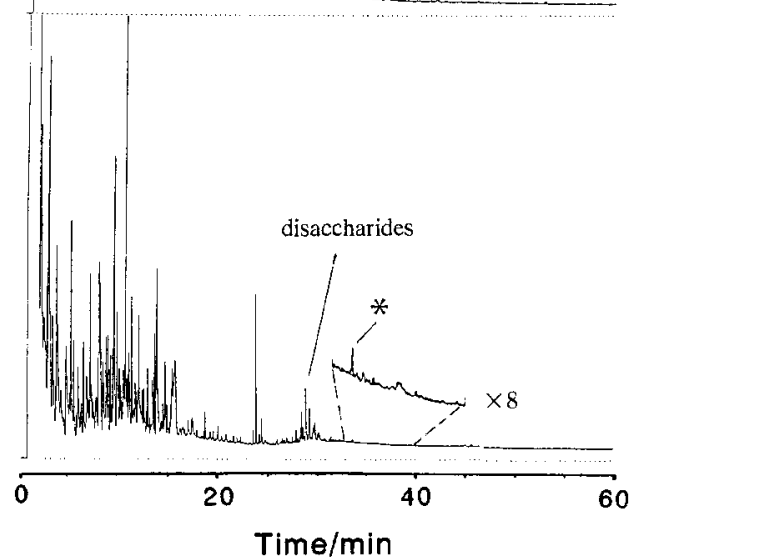

Fig. 3 Pyrograms of a paper sample (P-5) prepared by adding $1.0 \% \mathrm{~m} / \mathrm{m}$ rosin-glycerin ester at $400^{\circ} \mathrm{C}$ (a) without TMAH and (b) with TMAH, and (c) the blank paper sample (P-1) with TMAH. * This peak refers to one of the pyrolytic components of paper.

some pulp materials also contain the naturally occurring rosin acid to some extent. Therefore, in this work, the rosin glycerin ester sizing agents in paper samples were determined on the basis of only the two peaks of the methyl esters of the rosin adducts observed in the pyrogram.

\section{Determination of rosin-glycerin ester sizing agent in paper samples}

Figure 4 shows the relationship between the observed peak intensity of the methyl esters of the rosin adducts per sample weight (counts/ $\mu \mathrm{g}$ ) and the content of the sizing agents in standard paper samples. The thusobtained calibration curve exhibits very good linearity with $r=0.999$. Thus, the retained amounts of the sizing agent added to pulp slurry during the paper preparation were evaluated using this calibration curve.

Figure 5 shows the relationship between the amount of the retained sizing agents estimated by this method and the amount of the sizing agents added to the pulp slurry. It is interesting to note that all of the observed data fall below the octagonal broken line, which repre- 


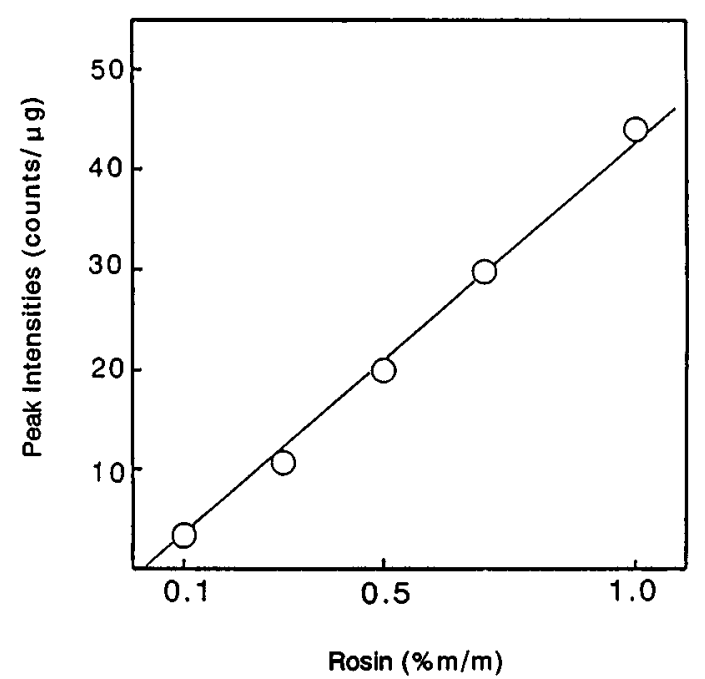

Fig. 4 Relationship between the amounts of the sizing agent added in the standard samples and the peak intensities of methyl esters of rosin adducts in the pyrograms.

sents complete retention (100\%), because a part of the sizing agent added into the pulp slurry is lost with the white water. These data suggest that about $50 \%$ of the sizing agents added into the pulp slurry are retained in this particular case, regardless of the added amount of the sizing agent. The observed reproducibility of this method is within $3 \%$ of the relative standard deviation for three repetitive runs with the same sample (P-5). Thus, high resolution Py-GC combined with reactive pyrolysis proved to be a powerful method for the rapid, convenient and precise analysis of the rosin-glycerin ester sizing agents in paper samples.

Financial support by the Grant-in-aid for Scientific Research (A)(09305056) and (09555262) of the Ministry of Education, Science, Sports and Culture, Japan, and by a Grant from the "Research for the Future" Program of the Japan Society for the

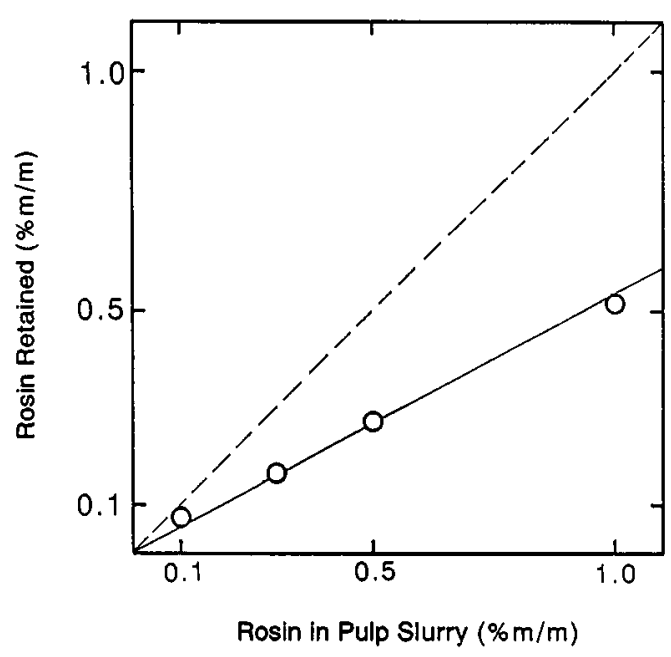

Fig. 5 Relationship between the amounts of the sizing agent added into a pulp slurry and those retained in paper samples estimated by PyGC.

Promotion of Science (JSPS-RFTF, 96R11601) are gratefully acknowledged.

\section{References}

1. TAPPI Test Method T 408 om-82,"Rosin in paper and paperboard".

2. Y. Sumikawa, Y. Kishika and H. Toda, Japan Tappi J., 22, 1400 (1968).

3. T. Yano, H. Ohtani, S. Tsuge and T. Obokato, Tappi J., 74, 197 (1991).

4. T. Yano, H. Ohtani, S. Tsuge and T. Obokato, Analyst [London], 117, 849 (1992).

5. Y. Ishida, H. Ohtani, T. Kato, S. Tsuge and T. Yano, Tappi J., 77, 177 (1994). 